\title{
MicroRNA-138 inhibits migration and invasion of non-small cell lung cancer cells by targeting LIMK1
}

\author{
YANJUAN TAN ${ }^{1}$, HUAIDONG HU ${ }^{2}$, WUYUAN TAN ${ }^{3}$, LONGYU JIN ${ }^{2}$, JIANXIN LIU $^{2}$ and HUI ZHOU ${ }^{4,5}$ \\ ${ }^{1}$ Neonatal Intensive Care Unit; ${ }^{2}$ Department of Cardiothoracic Surgery, The Third Xiangya Hospital of Central South University, \\ Changsha, Hunan 410013; ${ }^{3}$ Department of Burns, Xiangya Hospital of Central South University, Changsha, Hunan 410078; \\ ${ }^{4}$ Department of Medical Oncology, Tumor Hospital of Hunan, Changsha, Hunan 410000; \\ ${ }^{5}$ State Key Laboratory of Medical Genetics, Central South University, Changsha, Hunan 410078, P.R. China
}

Received April 4, 2015; Accepted February 15, 2016

DOI: $10.3892 / \mathrm{mmr} .2016 .5769$

\begin{abstract}
MicroRNA (miR)-138 has previously been demonstrated to have a suppressive role in numerous types of human cancer, including non-small cell lung cancer (NSCLC). LIM domain kinase 1 (LIMK1) is a serine/threonine kinase that regulates actin polymerization via phosphorylation and inactivation of cofilin. Previous studies have reported that LIMK1 is associated with NSCLC; however, the underlying regulatory mechanism of LIMK1, and the association between LIMK1 and miR-138 in NSCLC cells, remains largely unknown. The present study aimed to reveal the regulatory roles of miR-138 and LIMK1 in NSCLC cell migration and invasion. Reverse transcription-quantitative polymerase chain reaction and western blot analysis were used to examine the mRNA and protein expression levels. Transwell and wound healing assays were conducted to determine cell invasion and migration. A luciferase reporter assay was used to determine the target association between miR-138 and LIMK1. The present study demonstrated that miR-138 was markedly downregulated in NSCLC tissues and cell lines, whereas the expression levels of LIMK1 were significantly upregulated. LIMK1 was further identified as a direct target of miR-138 in NSCLC H460 cells. Furthermore, overexpression of miR-138 significantly inhibited the protein expression of LIMK1, whereas knockdown of miR-138 upregulated the protein expression of LIMK1 in $\mathrm{H} 460$ cells. In addition, overexpression of miR-138 significantly inhibited the migration and invasion of NSCLC cells; however, overexpression of LIMK1 significantly promoted NSCLC cell migration and invasion. An investigation into the underlying molecular mechanism revealed that overexpression of miR-138 significantly decreased cofilin signaling
\end{abstract}

Correspondence to: Dr Huaidong Hu, Department of Cardiothoracic Surgery, The Third Xiangya Hospital of Central South University, 138 Tongzipo Road, Changsha, Hunan 410013, P.R. China E-mail: csuhuhuaidong@163.com

Key words: non-small cell lung cancer, microRNA-138, LIM domain kinase 1, migration, invasion, cofilin activity, whereas knockdown of miR-138 notably enhanced cofilin signaling activity. In conclusion, the present study suggests that miR-138 may inhibit the migration and invasion of NSCLC cells by targeting the LIMK1/cofilin signaling pathway. Therefore, miR-138/LIMK1/cofilin may be considered a potential therapeutic target for the treatment of NSCLC.

\section{Introduction}

Lung cancer is the leading cause of cancer-associated mortality, the incidence of which is increasing worldwide. Non-small cell lung cancer (NSCLC) is the most common type of lung cancer. Despite improvements in diagnostic imaging, surgery, radiotherapy and chemotherapy, the overall survival rate for patients with NSCLC remains poor (1). Since human cancers, including NSCLC, have been reported to be associated with aberrant expression of oncogenes and tumor suppressors, investigations into the underlying molecular mechanisms may help develop novel therapeutic targets for NSCLC.

MicroRNAs (miRs) are small (18-25 nucleotides) non-coding RNA molecules, which have been demonstrated to exert suppressive effects on the regulation of gene expression at the post-transcriptional level. miRs are able to directly bind to the 3'-untranslational region (UTR) of their target mRNAs, either inhibiting their translation or inducing their degradation (2). In recent years, dysregulated miRs have been identified in various types of human cancer, including NSCLC. miR-138 generally acts as a tumor suppressor in malignant tumors, including glioblastoma (3), squamous cell carcinoma (4), ovarian cancer (5), and head and neck squamous cell carcinoma (6). Zhang et al (7) reported that miR-138 may inhibit tumor growth of NSCLC via targeting enhancer of zeste homolog 2 (EZH2). Yang et al (8) demonstrated that overexpression of miR-138 induced radiosensitization in lung cancer cells via targeting sentrin/SUMO-specific protease 1 (SENP1). Furthermore, pyruvate dehydrogenase kinase 1 and G protein-coupled receptor 124 (GPR124) have been identified as direct targets of miR-138 in NSCLC cells $(9,10)$. Since one miR may have several target genes (11), whether other target genes of miR-138 exist in NSCLC remains to be elucidated. 
As a serine-threonine protein kinase, LIM domain kinase 1 (LIMK1) has been reported to participate in actin polymerization and reorganization of the actin cytoskeleton via phosphorylation and inactivation of cofilin $(12,13)$. In addition, LIMK1 has been revealed to be frequently upregulated in several types of human cancer, where it has an oncogenic role $(14,15)$. Chen et al (14) demonstrated that inhibition of LIMK1 expression suppressed NSCLC cell migration and enhanced their sensitivity to chemotherapy drugs. However, the detailed role of LIMK1, and its regulatory mechanism, in NSCLC remains largely unknown.

The present study aimed to explore the molecular mechanism by which miR-138 mediates the migration and invasion of NSCLC cells, focusing on its potential target LIMK1.

\section{Materials and methods}

Materials and reagents. Fetal bovine serum (FBS), Dulbecco's phosphate-buffered saline (DPBS), TRIzol ${ }^{\circledR}$ reagent, Lipofectamine ${ }^{\circledR} 2000$, Cellfectin II Reagent, OPTI-MEM medium, and mirVana ${ }^{\mathrm{TM}}$ quantitative polymerase chain reaction (qPCR) miRNA Detection kit were purchased from Thermo Fisher Scientific, Inc. (Waltham, MA, USA). Standard SYBR-Green reverse transcription (RT)-PCR kit was purchased from Takara Bio. Inc. (Otsu, Japan). Quick-Change Site-Directed Mutagenesis kit was purchased from Agilent Technologies, Inc. (La Jolla, CA, USA). PsiCHECK ${ }^{\mathrm{TM}}$-2 vector and Dual-Luciferase Reporter Assay system were purchased from Promega Corporation (Madison, WI, USA). Transwell chambers (24-well) were purchased from Chemicon; EMD Millipore (Billerica, MD, USA). Bicinchoninic acid (BCA) Protein Assay kit and Enhanced chemiluminescence (ECL) Western Blotting kit were purchased from Pierce Biotechnology, Inc. (Rockford, IL, USA). All antibodies were purchased from Abcam (Cambridge, MA, USA).

Tissue specimen collection. The present study was approved by the Ethics Committee of Central South University (Changsha, China). Written informed consent was obtained from each patient. The NSCLC patients $(n=16)$ included 10 men and 6 women aged between 45 and 73 years old (mean age, 56.4 years). Patients were recruited between April 2013 and October 2013. All patients received neither radiation therapy nor chemotherapy prior to surgical resection. A total of 16 primary NSCLC tissues, and their matched adjacent normal tissues, were collected at the Department of Cardiothoracic Surgery, The Third Xiangya Hospital of Central South University (Changsha, China). The histomorphology of all samples was confirmed by the Department of Pathology, The Third Xiangya Hospital of Central South University. Tissues were immediately snap-frozen in liquid nitrogen following surgical removal and stored at $-80^{\circ} \mathrm{C}$ prior to use.

Cell lines and cell culture. The H460, SK-MES-1, A549 and SPC-A1 human NSCLC cell lines, and the BEAS-2B normal human lung epithelial cell line were purchased from the Cell Bank of Central South University (Changsha, China). All cells were cultured in Dulbecco's modified Eagles' medium (DMEM; Thermo Fisher Scientific, Inc.) supplemented with $10 \% \mathrm{FBS}$ at $37^{\circ} \mathrm{C}$ in an atmosphere containing $5 \% \mathrm{CO}_{2}$.
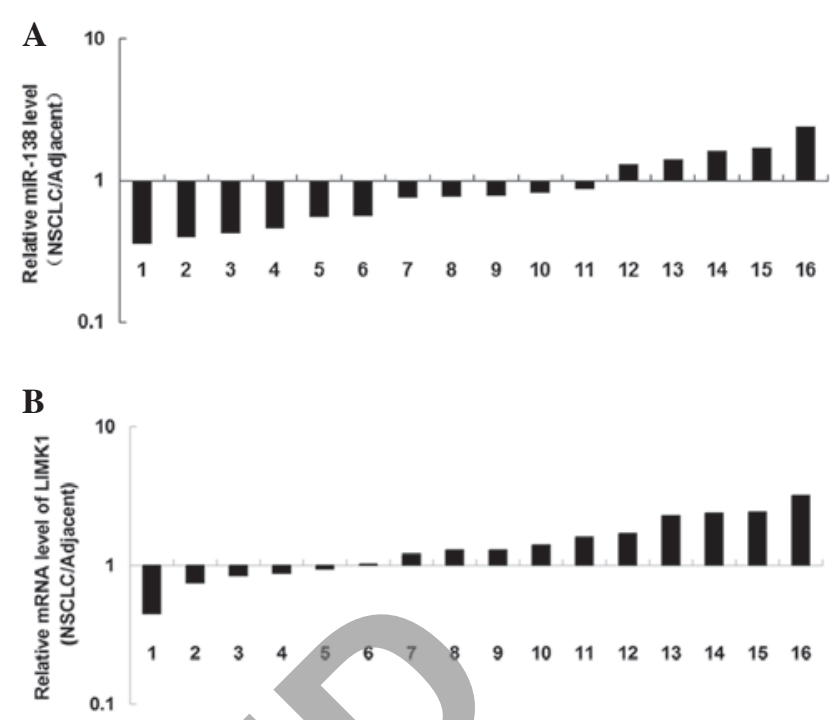

Figure 1. Reverse transcription-quantitative polymerase chain reaction was conducted to examine the expression levels of (A) microRNA (miR)-138 and (B) LIM domain kinase 1 (LIMK1) in 16 cases of non-small cell lung cancer samples (NSCLC) and their matched adjacent normal tissues.

RNA extraction and RT-qPCR. Tissues were homogenized and total RNA was extracted from the tissues and cells using TRIzol ${ }^{\circledR}$ reagent, according to the manufacturer's protocol. Takara PrimeScript ${ }^{\mathrm{TM}}$ RT-PCR kit (Takara Biotechnology Co., Ltd., Dalian, China) was used to perform the RT prior to mRNA and miRNA detection, according to the manufacturer's instruction. The relative expression levels of miR-138 were determined by RT-qPCR using mirVana ${ }^{\mathrm{TM}}$ qPCR microRNA Detection kit, according to the manufacturer's protocol. An Applied Biosystems ${ }^{\circledR} 7500$ thermal cycler, (Thermo Fisher Scientific, Inc.) was used and the reaction conditions were as follows: $95^{\circ} \mathrm{C}$ for $10 \mathrm{~min}, 40$ cycles at $95^{\circ} \mathrm{C}$ for $15 \mathrm{sec}$ and $60^{\circ} \mathrm{C}$ for $30 \mathrm{sec}$. For the PCR reaction, $1 \mu \mathrm{l}$ cDNA solution, $10 \mu \mathrm{l}$ PCR master mix, $2 \mu \mathrm{l}$ of primers and $7 \mu 1 \mathrm{H}_{2} \mathrm{O}$ were mixed to obtain a final reaction volume of $20 \mu \mathrm{l}$. Specific primer sets for miR-138 and U6 were obtained from Genecopoeia (Rockville, MD, USA). The mRNA expression levels of LIMK1 were detected by RT-qPCR using the standard SYBR-Green RT-PCR kit, according to the manufacturer's protocol. The specific primer pairs (purchased from Sangon Biotech Co., Ltd., Shanghai, China) were as follows: LIMK1, sense 5'-CAA GGGACTGGTTATGGTGGC-3', antisense 5'-CCCCGTCAC CGATAAAGGTC-3'; and $\beta$-actin (used as an internal control), sense 5'-AGGGGCCGGACTCGTCATACT-3', and antisense 5'-GGCGGCACCACCATGTACCCT-3'. The relative expression levels of LIMK1 mRNA or miR-138 were quantified using GraphPad Prism 4.0 software (GraphPad Software, Inc., San Diego, CA, USA), and the $2^{-\Delta \Delta \mathrm{Cq}}$ method (16).

Western blotting. Cells were lysed in cold radioimmunoprecipitation assay buffer (Thermo Fisher Scientific, Inc.). The BCA Protein Assay kit was used to determine protein concentration. Protein samples $(50 \mu \mathrm{g})$ were separated by $10 \%$ sodium dodecyl sulfate-polyacrylamide gel electrophoresis and were transferred to a polvinylidene difluoride (PVDF) membrane (Thermo Fisher Scientific, Inc.). The PVDF membrane was blocked with $5 \%$ nonfat dried milk in PBS for $4 \mathrm{~h}$. Subsequently, 

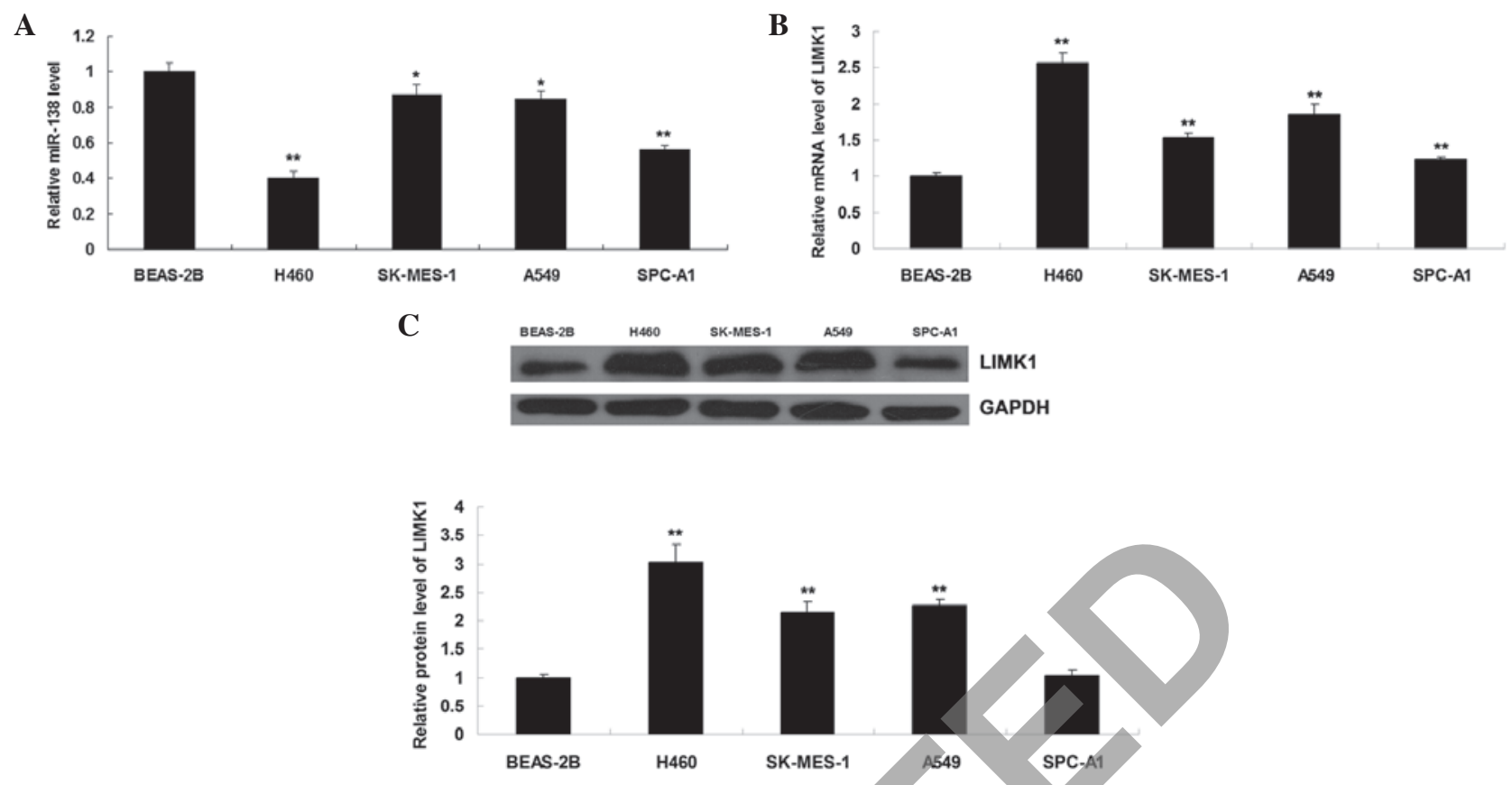

Figure 2. Reverse transcription-quantitative polymerase chain reaction (RT-qPCR) was conducted to examine the expression levels of (A) microRNA (miR)-138 in the following human non-small cell lung cancer (NSCLC) cell lines: H460, SK-MES-1, A549 and SPC-A1, as well as in the BEAS-2B normal human lung epithelial cell line. RT-qPCR and western blotting were conducted to determine the (B) mRNA and (C) protein expression levels of LIM domain kinase 1 (LIMK1) in NSCLC and normal human lung epithelial cell lines. Data are presented as the mean \pm standard deviation. ${ }^{*} \mathrm{P}<0.05,{ }^{* *} \mathrm{P}<0.01$ vs. BEAS-2B cells. GAPDH, glyceraldehyde 3-phosphate dehydrogenase.

the PVDF membrane was incubated with mouse anti-LIMK1 monoclonal antibody (1:200; cat no. ab117623), mouse anti-cofilin monoclonal antibody (1:200; cat no. ab54532), rabbit anti-phosphorylated-cofilin monoclonal antibody (1:200; cat no. ab47281) and mouse anti-glyceraldehyde 3-phosphate dehydrogenase (GAPDH) monoclonal antibody (1:50; ab8245) at room temperature for $3 \mathrm{~h}$. The membrane was washed three times with PBS ( $5 \mathrm{~min} / \mathrm{wash}$ ) and then incubated with rabbit anti-mouse secondary antibody (1:10,000; cat no. ab6728) or goat anti-rabbit secondary antibody (1:10,000 cat no. ab6721). Following a further three 5 min washes with DPBS, an ECL Western Blotting kit was used to detect the immune complexes on the PVDF membrane. Image-Pro Plus software 6.0 (Media Cybernetics, Inc., Rockville, MD, USA) was used to analyze relative protein expression levels, represented as the density ratio vs. GAPDH. GAPDH was used as an internal reference.

Transfection. LIMK1 plasmid, miR-138 mimics and a miR-138 inhibitor were generated by Nlunbio (Changsha, China). Blank pcDNA3.1(+) vector and scramble miRNA mimic were purchased from Nlunbio. Lipofectamine ${ }^{\circledR} 2000$ was used to perform transfection, according to the manufacturer's protocol. Briefly, LIMK1 plasmid, miRNA mimics or miR-138 inhibitor and Lipofectamine ${ }^{\circledR} 2000$ were diluted with serum-free medium. The diluted Lipofectamine ${ }^{\circledR} 2000$ was added to the diluted plasmid or miRNA mimics, and incubated for $20 \mathrm{~min}$ at room temperature. Subsequently, the mixture was added to the $\mathrm{H} 460$ cell suspension. The $\mathrm{H} 460$ cells were then incubated at $37^{\circ} \mathrm{C}$ in an atmosphere containing $5 \% \mathrm{CO}_{2}$ for $6 \mathrm{~h}$. Finally, the medium in each well was replaced with the normal serum-containing medium, and the cells were cultured for $24 \mathrm{~h}$ prior to the following assays.
Dual luciferase reporter assays. The normal and mutant (mut) 3'-UTRs of LIMK1 were constructed by PCR, and were then inserted into the multiple cloning site of the psiCHECK ${ }^{\mathrm{TM}}-2$ vector. For the luciferase reporter assay, $2 \times 10^{4} \mathrm{H} 460$ cells were cultured to $50-60 \%$ confluence in a 24-well plate. In each well, medium was replaced with $300 \mu$ I OPTI-MEM medium. The H460 cells were then co-transfected with psiCHECK ${ }^{\mathrm{TM}}-2$-LIMK1-3'-UTR or psiCHECK $^{\mathrm{TM}}$-2-LIMK1-mut 3'-UTR vector (both purchased from Nlunbio) plus $50 \mathrm{nM}$ miR-138 mimics using Cellfectin II reagent, according to the manufacturer's protocol. Cells were incubated with the transfection reagent/DNA complex for $5 \mathrm{~h}$, and the medium was then replaced with fresh complete medium. A Dual-Luciferase Reporter Assay system was used to determine the luciferase activities $48 \mathrm{~h}$ post-transfection using a Lucetta ${ }^{\mathrm{TM}}$ Luminometer, (cat no. AAL-1001; Lonza Group, Ltd., Basel, Switzerland). Renilla luciferase activity was normalized to firefly luciferase activity.

Wound healing assay. Cell migratory capability was estimated using a wound healing assay. Briefly, H460 cells were cultured to confluence. Wounds $(\sim 1 \mathrm{~mm})$ were created in the cell monolayer using a plastic scriber, and cells were washed and incubated in serum-free medium. A total of $24 \mathrm{~h}$ after wound generation, the cells were incubated in medium supplemented with $10 \%$ FBS. Cultures at 0 and $36 \mathrm{~h}$ were fixed and observed under a CX23 Microscope (Olympus Corporation, Tokyo, Japan).

Transwell assay. The invasive ability of H460 cells was determined in 24-well Transwell chambers, which contained a layer of Matrigel (BD Biosciences, Franklin Lakes, NJ, USA). 

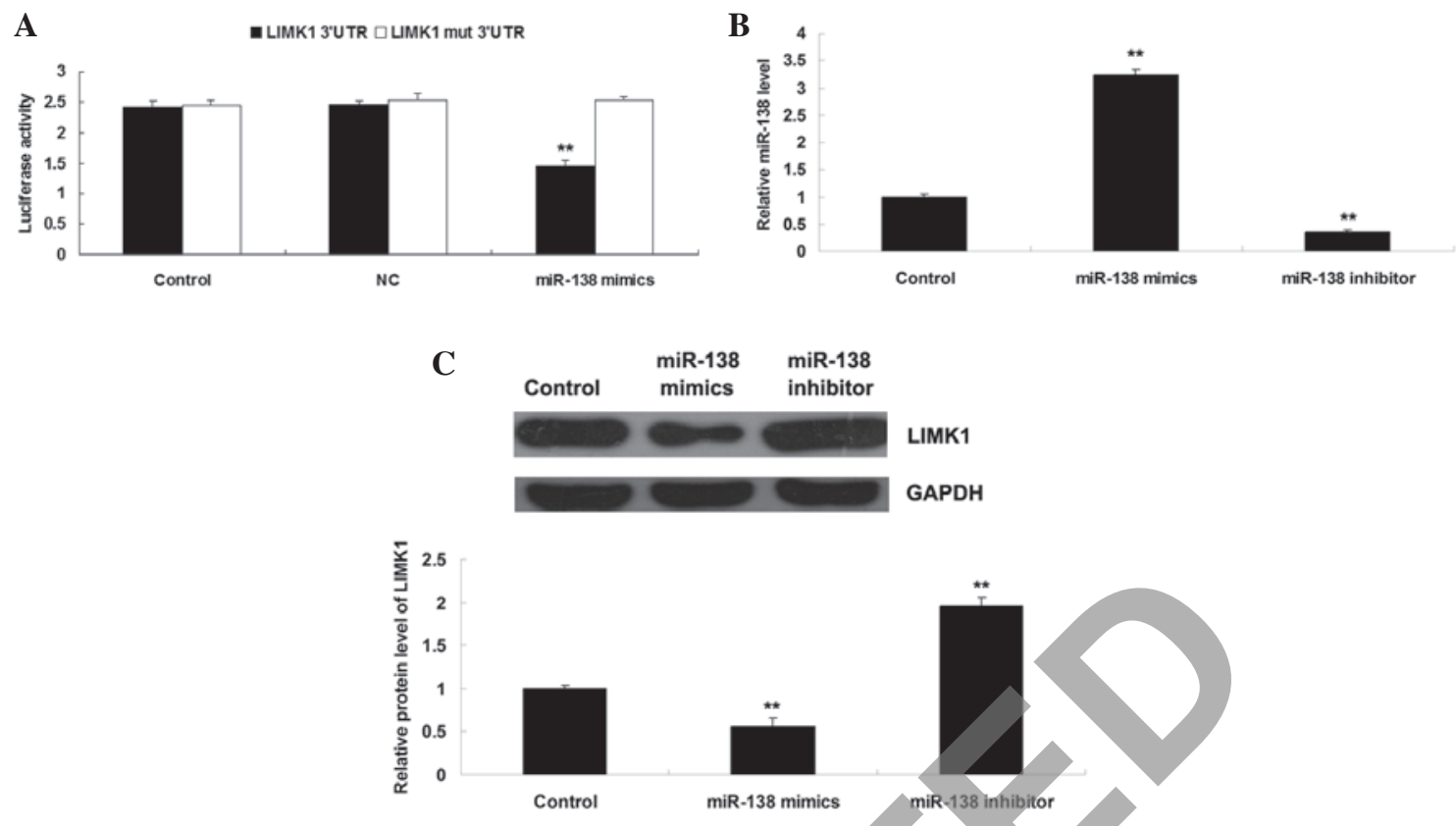

Figure 3. (A) H460 cells were co-transfected with psiCHECK ${ }^{\mathrm{TM}}$-2-LIM domain kinase 1 (LIMK1)-3'-untranslated region (UTR) or psiCHECK ${ }^{\mathrm{TM}}$-2-LIMK1-mut 3'-UTR vector plus microRNA (miR)-138 mimics or scramble miR mimics, as a negative control (NC). Dual-Luciferase Reporter Assay system was used to determine the luciferase activities $48 \mathrm{~h}$ post-transfection. Renilla luciferase activity was normalized to firefly luciferase activity. (B) Reverse transcription-quantitative polymerase chain reaction was conducted to examine the expression levels of miR-138 in H460 cells transfected with miR-138 mimics or a miR-138 inhibitor. (C) Western blotting was conducted to examine the protein expression levels of LIMK1 in H460 cellz transfected with miR-138 mimics or a miR-138 inhibitor. Untransfected cells were used as control. Data are presented as the mean \pm standard deviation. ${ }^{* *} \mathrm{P}<0.01$ vs. the control cells. GAPDH, glyceraldehyde 3-phosphate dehydrogenase.

For each group, a cell suspension $\left(1 \times 10^{5}\right.$ cells/ml $)$ was added to the upper chamber, whereas DMEM containing 10\% FBS was added to the lower chamber. Following a $24 \mathrm{~h}$ incubation, non-invading cells and the Matrigel on the interior of the inserts was removed using a cotton-tipped swab. Invasive cells on the lower surface of the membrane were stained with gentian violet, rinsed with water, and air-dried. Five fields were randomly selected and the cell number was counted under a CX23 Microscope (Olympus Corporation).

Statistical analysis. The results are presented as the mean \pm standard deviation of at least three independent experiments. Statistical analysis was performed using SPSS 17 software (SPSS Inc., Chicago, IL, USA). Statistical analysis of differences between groups was performed by one-way analysis of variance. $\mathrm{P}<0.05$ was considered to indicate a statistically significant difference.

\section{Results}

miR-138 is downregulated and LIMK1 is upregulated in NSCLC tissues and cell lines. To determine the role of miR-138 and LIMK1 in NSCLC, the present study examined the expression levels of miR-138 in human NSCLC tissues and matched adjacent normal tissues using RT-qPCR. As shown in Fig. 1A, miR-138 was significantly downregulated in NSCLC tissues compared with in the matched adjacent normal tissues. The mRNA expression levels of LIMK1 were also examined by RT-qPCR. As shown in Fig. 1B, the mRNA expression levels of LIMK1 were increased in NSCLC tissues, as compared with in the matched adjacent normal tissues. These results indicate that miR-138 is downregulated, whereas LIMK1 is upregulated in NSCLC tissues.

The present study further determined the expression levels of miR-138 in the following human NSCLC cell lines: H460, SK-MES-1, A549 and SPC-A1. The BEAS-2B normal human lung epithelial cell line was used as a control. As shown in Fig. 2A, the expression levels of miR-138 were significantly reduced in the NSCLC cell lines compared with in BEAS-2B cells. Consistent with results of the tissue analysis, the mRNA and protein expression levels of LIMK1 were also increased in NSCLC cell lines compared with in BEAS-2B normal human lung epithelial cells (Fig. 2B and C). Since H460 cells exhibited the most significant changes in miR-138 and LIMK1 expression among these NSCLC cell lines, this cell line was used for subsequent experiments.

LIMK1 is a direct target of miR-138 in NSCLC cells. Bioinformatic prediction using Targetscan online software version 3.1 (targetscan.org) suggested that LIMK1 may be a direct target of miR-138. Therefore, the present study performed a luciferase reporter assay to confirm this relationship in NSCLC cells. As shown in Fig. 3A, luciferase activity was significantly reduced in H460 NSCLC cells co-transfected with miR-138 mimics and wild type LIMK1 3'-UTR. Conversely, luciferase activity was unchanged in the other groups compared with the control group (Fig. 3A). These results indicate that LIMK1 may be a direct target of miR-138 in NSCLC cells.

Accordingly, the present study examined the role of miR-138 in the regulation of LIMK1 expression in NSCLC cells. Post-transfection with miR-138 mimics or a miR-138 
A

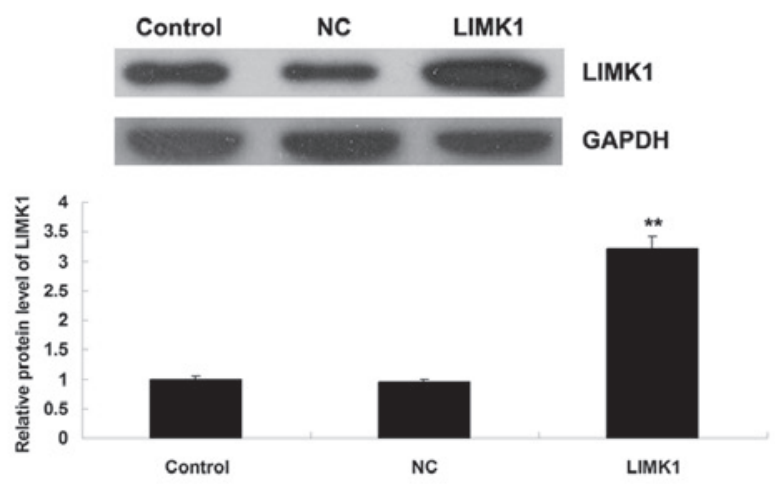

B
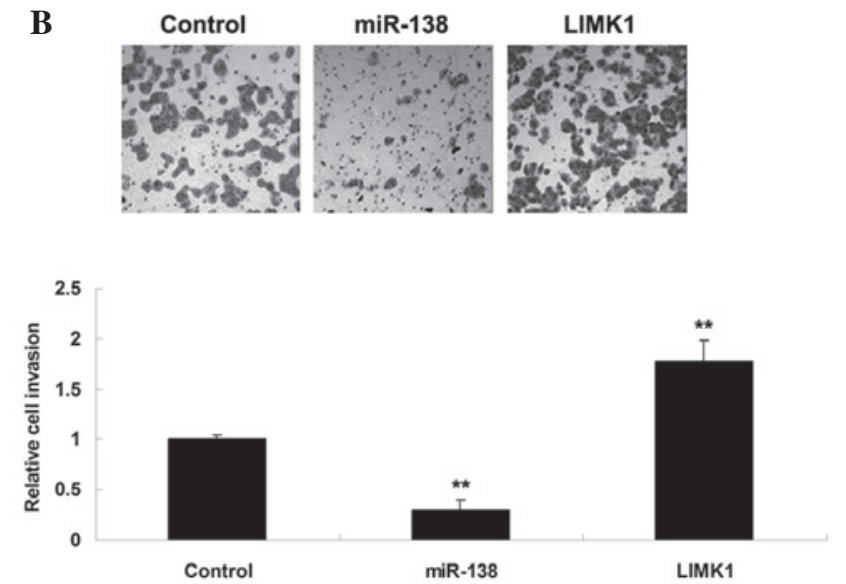

C

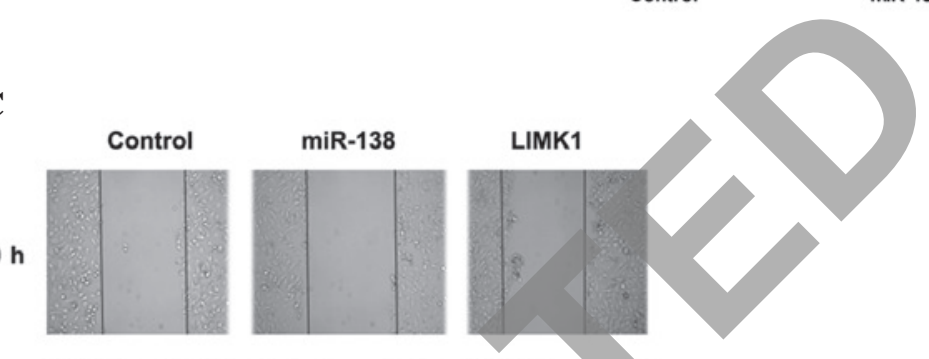

$36 \mathbf{h}$
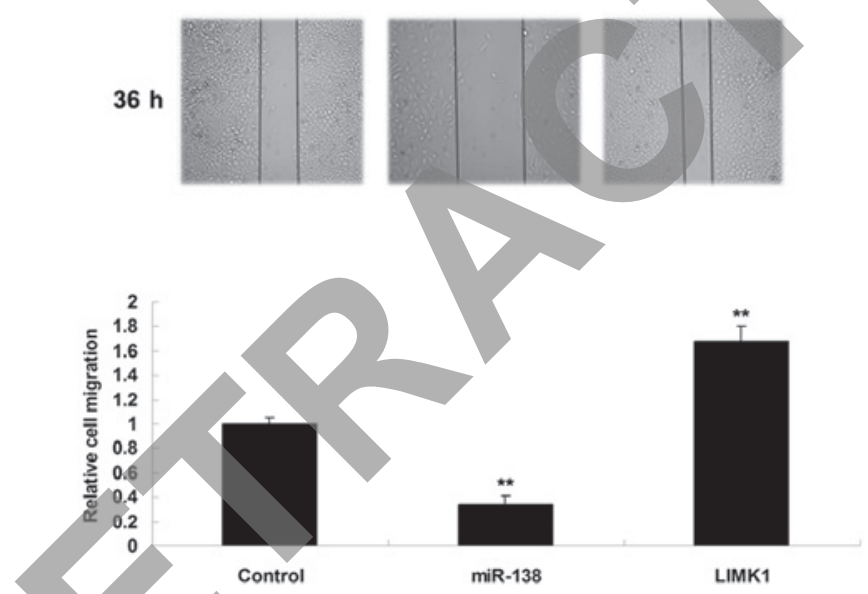

Figure 4. (A) Western blotting was conducted to examine the protein expression levels of LIM domain kinase 1 (LIMK1) in H460 cells transfected with a LIMK1 plasmid, or a blank vector as negative control (NC). Transwell and wound healing assays were conducted to determine the (B) invasion (magnification, x200) and (C) migration of H460 cells overexpressing miR-138 or LIMK1 (magnification, x40). Untransfected cells were used as a control. Data are presented as the mean \pm standard deviation. ${ }^{* *} \mathrm{P}<0.01$ vs. the control. GAPDH, glyceraldehyde 3 -phosphate dehydrogenase.

inhibitor, the expression levels of miR-138 were detected. As shown in Fig. 3B, H460 cells transfected with miR-138 mimics exhibited a significant increase in miR-138 expression, whereas transfection with the miR-138 inhibitor markedly suppressed miR-138 expression, as compared with the control group. These results indicate that the transfection was successful. Since miRs can negatively regulate the expression of their target genes at the post-transcriptional level, the present study subsequently detected the protein expression levels of LIMK1 in each group. As shown in Fig. 3C, the protein expression levels of LIMK1 were reduced in H460 NSCLC cells post-transfection with miR-138 mimics, but were increased following miR-138 knockdown compared with in the control group. These findings further confirm that miR-138 may negatively regulate the expression of LIMK1 via directly binding to the 3'-UTR of its mRNA in H460 NSCLC cells.
miR-138 inhibits NSCLC cell invasion and migration by targeting LIMK1. The roles of LIMK1 and miR-138 in NSCLC cell migration and invasion were further studied. As well as modulating miR-138 expression in H460 cells, the present study also transfected H460 cells with a LIMK1 plasmid, in order to upregulate its expression. As shown in Fig. 4A, transfection with the LIMK1 plasmid markedly enhanced the protein expression levels of LIMK1 compared with in the control group. Subsequently, the roles of miR-138 and LIMK1 in the invasion of H460 NSCLC cells were investigated. Overexpression of miR-138 inhibited H460 NSCLC cell invasion, whereas overexpression of LIMK1 markedly promoted the invasion of H460 cells (Fig. 4B). In addition, overexpression of miR-138 led to a significant reduction in the migration of H460 NSCLC cells. Conversely, overexpression of LIMK1 significantly promoted the migration of H460 NSCLC cells (Fig. 4C). These results suggest that the 

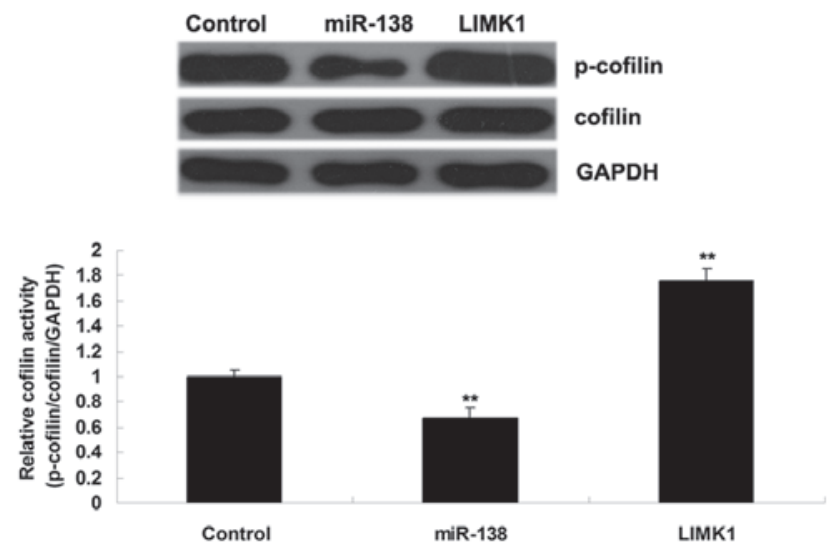

Figure 5. Western blotting was conducted to detect the protein expression levels of phosphorylated (p)-cofilin and total cofilin in H460 cells transfected with microRNA (miR)-138 mimic or LIM domain kinase 1 (LIMK1) expression plasmid. Glyceraldehyde 3-phosphate dehydrogenase (GAPDH) was used as an internal reference. Untransfected cells were used as a control. Data are presented as the mean \pm standard deviation. ${ }^{* *} \mathrm{P}<0.01$ vs. the control cells.

inhibitory effects of miR-138 on H460 NSCLC cell invasion and migration may be caused by direct inhibition of LIMK1 expression.

Downstream cofilin signaling is mediated by $\mathrm{miR}-138$ in NSCLC cells. It has been suggested that cofilin signaling, downstream of LIMK1, has an important role in mediating the migration and invasion of cancer cells. Therefore, the present study further examined the activity of this signaling pathway in NSCLC cells transfected with miR-138 mimics or a miR-138 inhibitor. As shown in Fig. 5, overexpression of miR-138 significantly inhibited the activity of cofilin, whereas inhibition of miR-138 promoted its activity in H460 NSCLC cells. These findings suggest that the inhibitory effects of miR-138 on H460 NSCLC cell migration and invasion may be caused by suppression of LIMK1/cofilin signaling activity.

\section{Discussion}

miR-138 has been demonstrated to have a suppressive role in numerous types of human cancer $(4-6,17,18)$. The present study revealed that miR-138 was downregulated in NSCLC tissues and cell lines, accompanied with the upregulation of LIMK1, a target gene of miR-138. Furthermore, miR-138 negatively mediated the protein expression of LIMK1 in NSCLC cells. Overexpression of miR-138 significantly inhibited the migration and invasion of NSCLC cells, whereas overexpression of LIMK1 significantly promoted NSCLC cell migration and invasion. An investigation into the underlying molecular mechanism indicated that LIMK1/cofilin signaling was mediated by miR-138 in NSCLC cells. Therefore, these results suggested that miR-138 may inhibit the migration and invasion of NSCLC cells by targeting the LIMK1/cofilin signaling pathway.

miRs have been reported to have crucial roles in the development and progression of NSCLC (9,19-21). For example, miR-145 inhibits NSCLC cell proliferation by targeting c-Myc (22). In addition, miR-31 inhibits cisplatin-induced apoptosis in NSCLC cells by targeting the drug transporter ATP-binding cassette, sub-family B, member 9 (23). miR-148a suppresses epithelial-to-mesenchymal transition by targeting Rho-associated protein kinase 1 in NSCLC cells (24). Previous studies have also reported that miR-138 is frequently downregulated in NSCLC tissues and cells $(7,8)$. In the present study, the expression levels of miR-138 were significantly reduced in NSCLC tissues and cells, as compared with in normal adjacent tissues and a normal lung epithelial cell line, respectively. These results are consistent with previous findings $(7,8)$.

miR-138 has been implicated to have a role in $\operatorname{NSCLC}(8,9)$. For example, miR-138 inhibits proliferation of NSCLC cells by targeting 3-phosphoinositide-dependent protein kinase-1 and EZH2 (7,10). Gao et al (9) demonstrated that miR-138-5p reversed gefitinib resistance in NSCLC cells via inhibition of GPR124. In addition, Yang et al (8) revealed that high levels of miR-138 were associated with reduced lung cancer cell proliferation and colony formation, and suggested that miR-138 was associated with radiosensitization in lung cancer cells by targeting SENP1. However, the role of miR-138 in the regulation of NSCLC metastasis remains largely unknown. The present study further demonstrated that miR-138 had a suppressive role in the regulation of migration and invasion of H460 NSCLC cells, thus suggesting that miR-138 may inhibit the metastasis of NSCLC.

The results of the present study demonstrated that LIMK1, a target of miR-138, was involved in the miR-138-mediated migration and invasion of NSCLC cells. LIMK1 is a serine/threonine kinase, which belongs to a small subfamily that contain a unique combination of $2 \mathrm{~N}$-terminal LIM motifs and a C-terminal protein kinase domain (12). The oncogenic role of LIMK1 has been reported in several types of human cancer (14,17). For example, Zhang et al (25) reported that LIMK1 was able to promote the migratory ability of multidrug-resistant osteosarcoma cells. Tapia et al (26) demonstrated that LIMK1 had a role in the regulation of prostate cancer cell invasion by modulating the function of membrane-type matrix metalloproteinase 1. Furthermore, LIMK1 has been shown to enhance the progression of human breast cancer (27). The present study revealed that LIMK1 was upregulated in NSCLC tissues and cell lines, thus suggesting an oncogenic role. These results were consistent with those of a previous study, which reported that LIMK1 was frequently upregulated in lung tissues, and overexpression of LIMK1 was associated with high tumor-node-metastasis stage and lymph node metastasis in patients with NSCLC (14). In addition, knockdown of LIMK1 expression may markedly inhibit migration and invasion of 801D lung cancer cells, and sensitize $801 \mathrm{D}$ cells to chemotherapeutic drugs cisplatin and gemcitabine (14). The present study demonstrated that overexpression of LIMK1 markedly promoted the migration and invasion of H460 NSCLC cells. In addition, LIMK1 has been reported to be mediated by other miRs in NSCLC. For example, miR-27b inhibits the growth and invasion of NSCLC cells by targeting LIMK1 (15).

LIMK1 has previously been reported to regulate actin polymerization via phosphorylation and inactivation of the actin binding factor cofilin (13). The present study demonstrated that the downstream cofilin signaling pathway was mediated by miR-138 in NSCLC cells. In the present study, 
overexpression of miR-138 significantly inhibited the activity of cofilin, whereas inhibition of miR-138 promoted its activity in H460 NSCLC cells, thus suggesting that the inhibitory effects of miR-138 on NSCLC cell migration and invasion may be realized by suppressing LIMK1/cofilin signaling activity.

In conclusion, the present study is the first, to the best of our knowledge, to suggest that miR-138 has an inhibitory role in the regulation of NSCLC cell migration and invasion by targeting the LIMK1/cofilin signaling pathway. Therefore, miR-138/LIMK1/cofilin may be considered a potential therapeutic target for the treatment of NSCLC.

\section{Acknowledgements}

The present study was supported by the Natural Science Foundation of Hunan Province (grant no. 2015JJ6058).

\section{References}

1. Greenlee RT, Murray T, Bolden S and Wingo PA: Cancer statistics, 2000. CA Cancer J Clin 50: 7-33, 2000.

2. Huang X, Liang M, Dittmar R and Wang L: Extracellular microRNAs in urologic malignancies: Chances and challenges. Int J Mol Sci 14: 14785-14799, 2013.

3. Qiu S, Huang D, Yin D, Li F, Li X, Kung HF and Peng Y: Suppression of tumorigenicity by microRNA-138 through inhibition of EZH2-CDK4/6-pRb-E2F1 signal loop in glioblastoma multiforme. Biochim Biophys Acta 1832: 1697-1707, 2013.

4. Liu X, Wang C, Chen Z, Jin Y, Wang Y, Kolokythas A, Dai Y and Zhou X: MicroRNA-138 suppresses epithelial-mesenchyma transition in squamous cell carcinoma cell lines. Biochem $J 440$ 23-31, 2011.

5. Yeh YM, Chuang CM, Chao KC and Wang LH: MicroRNA-138 suppresses ovarian cancer cell invasion and metastasis by targeting SOX4 and HIF-1 $\alpha$. Int J Cancer 133: 867-878, 2013.

6. Liu X, Jiang L, Wang A, Yu J, Shi F and Zhou X: MicroRNA-138 suppresses invasion and promotes apoptosis in head and neck squamous cell carcinoma cell lines. Cancer Lett 286: 217-222, 2009.

7. Zhang H, Zhang H, Zhao M, Lv Z, Zhang X, Qin X, Wang H, Wang S, Su J, Lv X, et al: MiR-138 inhibits tumor growth through repression of EZH2 in non-small cell lung cancer. Cell Physiol Biochem 31: 56-65, 2013.

8. Yang H, Tang Y, Guo W, Du Y, Wang Y, Li P, Zang W, Yin X, Wang $\mathrm{H}$, Chu $\mathrm{H}$, et al: Up-regulation of microRNA-138 induce radiosensitization in lung cancer cells. Tumour Biol 35: 6557-6565, 2014.

9. Gao Y, Fan X, Li W, Ping W, Deng Y and Fu X: miR-138-5p reverses gefitinib resistance in non-small cell lung cancer cells via negatively regulating $\mathrm{G}$ protein-coupled receptor 124 . Biochem Biophys Res Commun 446: 179-186, 2014.

10. Ye XW, Yu H, Jin YK, Jing $X T, X u M$, Wan $Z F$ and Zhang XY: miR-138 inhibits proliferation by targeting 3 -phosphoinositide-dependent protein kinase-1 in non-small cell lung cancer cells. Clin Respir J 9: 27-33, 2015.
11. Liu X, Fortin K and Mourelatos Z: MicroRNAs: Biogenesis and molecular functions. Brain Pathol 18: 113-121, 2008.

12. te Velthuis AJ and Bagowski CP: PDZ and LIM domain-encoding genes: Molecular interactions and their role in development. Scientific World Journal 7: 1470-1492, 2007.

13. Bernard O: Lim kinases, regulators of actin dynamics. Int J Biochem Cell Biol 39: 1071-1076, 2007.

14. Chen Q, Jiao D, Hu H, Song J, Yan J, Wu L and Xu LQ: Downregulation of LIMK1 level inhibits migration of lung cancer cells and enhances sensitivity to chemotherapy drugs. Oncol Res 20: 491-498, 2013.

15. Wan L, Zhang L, Fan K and Wang J: MiR-27b targets LIMK1 to inhibit growth and invasion of NSCLC cells. Mol Cell Biochem 390: 85-91, 2014.

16. Livak KJ and Schmittgen TD: Analysis of relative gene expression data using real-time quantitative PCR and the 2(-Delta Delta C(T)) Method. Methods 25: 402-408, 2001.

17. Chen P,Zeng M, Zhao Y and Fang X: Upregulation of Limk1 caused by microRNA-138 loss aggravates the metastasis of ovarian cancer by activation of Limk1/cofilin signaling. Oncol Rep 32: 2070-2076, 2014.

18. Gong H, Song L, Lin C, Liu A, Lin X, Wu J, Li M and Li J: Downregulation of miR-138 sustains NF- $\kappa$ B activation and promotes lipid raft formation in esophageal squamous cell carcinoma. Clin Cancer Res 19: 1083-1093, 2013.

19. Liu Y, Li M, Zhang G and Pang Z: MicroRNA-10b overexpression promotes non-small cell lung cancer cell proliferation and invasion. Eur J Med Res 18: 41, 2013.

20. Wang H, Li M, Zhang R, Wang Y, Zang W, Ma Y, Zhao G and Zhang G: Effect of miR-335 upregulation on the apoptosis and invasion of lung cancer cell A549 and H1299. Tumour Biol 34: 3101-3109, 2013

21. Zhang JG, Guo JF, Liu DL, Liu Q and Wang JJ: MicroRNA-101 exerts tumor-suppressive functions in non-small cell lung cancer through directly targeting enhancer of zeste homolog 2 . J Thorac Oncol 6: 671-678, 2011.

22. Chen Z, Zeng H, Guo Y, Liu P, Pan H, Deng A and Hu J: miRNA-145 inhibits non-small cell lung cancer cell proliferation by targeting c-Myc. J Exp Clin Cancer Res 29: 151, 2010.

23. Dong Z, Zhong Z, Yang L, Wang S and Gong Z: MicroRNA-31 inhibits cisplatin-induced apoptosis in non-small cell lung cancer cells by regulating the drug transporter ABCB9. Cancer Lett 343: 249-257, 2014.

24. Li J, Song Y, Wang Y, Luo J and Yu W: MicroRNA-148a suppresses epithelial-to-mesenchymal transition by targeting ROCK1 in non-small cell lung cancer cells. Mol Cell Biochem 380: 277-282, 2013.

25. Zhang H, Wang Y, Xing F, Wang J, Wang Y, Wang H, Yang Y and Gao Z: Overexpression of LIMK1 promotes migration ability of multidrug-resistant osteosarcoma cells. Oncol Res 19: 501-509, 2011.

26. Tapia T, Ottman $\mathrm{R}$ and Chakrabarti R: LIM kinase1 modulates function of membrane type matrix metalloproteinase 1: Implication in invasion of prostate cancer cells. Mol Cancer 10: 6, 2011.

27. McConnell BV, Koto K and Gutierrez-Hartmann A: Nuclear and cytoplasmic LIMK1 enhances human breast cancer progression. Mol Cancer 10: 75, 2011. 